\title{
Blood Group Antigen Matching Influence on \\ Gestational Outcomes (AMIGO) Study
}

Meghan Delaney ${ }^{1}$, Agneta Wikman $^{2}$, Leo van de Watering ${ }^{3}$, Henk Schonewille ${ }^{3}$, Jennie P. Verdoes ${ }^{3}$, Stephen P. Emery ${ }^{4}$, Michael F Murphy ${ }^{5}$, Julie Staves ${ }^{6}$, Susanne Flach ${ }^{6}$, Donald M. Arnold $^{7}$, Richard M Kaufman ${ }^{8}$, Alyssa Ziman ${ }^{9}$, Sarah K Harm ${ }^{10}$, Mark Fung ${ }^{10}$, Catherine S Eppes $^{11}$, Nancy M Dunbar ${ }^{12}$, Andreas Buser ${ }^{13}$, Erin Meyer ${ }^{14}$, Helen Savoia ${ }^{15}$, Padmakumari Abeysinghe $^{15}$, Nancy Heddle ${ }^{7}$, Alan Tinmouth ${ }^{16}$, Aicha N. Traore ${ }^{7}$, Mark H. Yazer ${ }^{4}$, for the BEST Collaborative

1. Bloodworks Northwest \& Department of Laboratory Medicine \& Pediatrics, University of Washington, Seattle, WA, USA

2. Clinical Immunology and Transfusion Medicine, Karolinska University Hospital and Laboratory Medicine, Karolinska Institutet, Stockholm, Sweden

3. Sanquin - Leiden University Medical Centre, Center for Clinical Transfusion Research, Leiden, Netherlands

4. University of Pittsburgh School of Medicine, and the Institute for Transfusion Medicine, Pittsburgh, PA, USA

5. NHS Blood and Transplant, Oxford University Hospitals and National Institute for Health Research (NIHR) Oxford Biomedical Research Centre, Oxford, UK

6. Department of Obstetrics, Gynecology, and Reproductive Sciences, Oxford University Hospitals, Oxford, UK

7. McMaster University, Hamilton, Ontario, Canada

8. Division of Transfusion Medicine, Department of Pathology, Brigham and Women's Hospital, Boston, MA, USA

9. Division of Transfusion Medicine, Department of Pathology and Laboratory Medicine, David Geffen School of Medicine at UCLA, Los Angeles, CA, USA

10. Department of Pathology and Laboratory Medicine, University of Vermont Medical Center, Burlington, VT, USA

11. Department of Maternal Fetal Medicine, Baylor College of Medicine, Houston, TX, USA

12. Department of Pathology and Department of Medicine, Dartmouth-Hitchcock Medical Center, Lebanon, $\mathrm{NH}$, USA

13. Regional Blood Transfusion Service, Swiss Red Cross, Basel, Switzerland

14. Department of Hematology/Oncology \& Pathology, Nationwide Children's Hospital, Columbus, $\mathrm{OH}, \mathrm{USA}$

15. Department of Hematology, The Royal Children's Hospital, Melbourne, Australia

16. Departments of Medicine, and Laboratory Medicine \& Pathology, University of Ottawa; University of Ottawa Centre for Transfusion Research, Ottawa Hospital Research Institute, Ottawa, Canada

\section{Corresponding author:}

Meghan Delaney, DO, MPH

Bloodworks Northwest

Department of Laboratory Medicine \& Paediatrics, University of Washington

921 Terry Avenue, Seattle, WA 98104, USA

Email: meghand@bloodworksnw.org

Phone: 2066896510

This is the author manuscript accepted for publication and has undergone full peer review but has not been through the copyediting, typesetting, pagination and proofreading process, which may lead to differences between this version and the Version record. Please cite this article as doi:10.1111/ trf.13977. 
Conflicts: None

Word count: 2954

Key words: Hemolytic disease of the fetus and newborn, blood groups, transfusion, obstetrics, alloimmunization

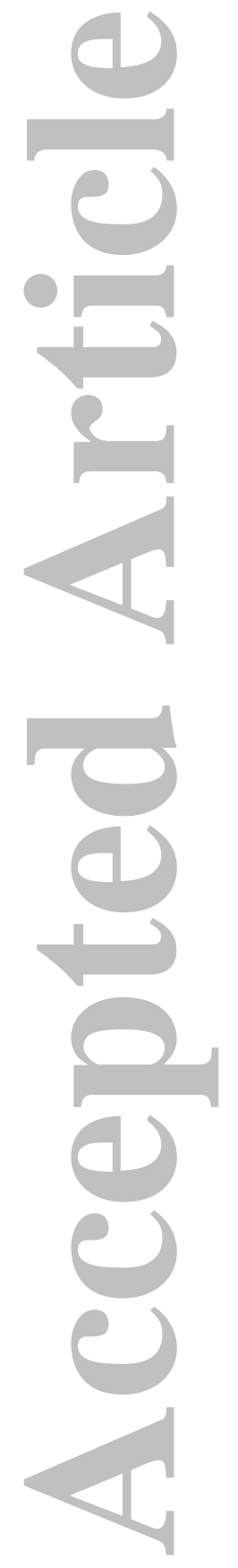

ScholarOne, 375 Greenbrier Drive, Charlottesville, VA, 229011 (434) 964-4100

This article is protected by copyright. All rights reserved. 


\section{ABSTRACT}

Background: Red blood cell (RBC) antigen matching policies to prevent alloimmunization in females of childbearing potential (FCP) vary between centers. To inform transfusion centers responsible for making decisions about matching policies for FCPs, the causal stimulus of the antibodies implicated in severe HDFN must be determined.

Methods: We conducted a multi-national retrospective study of women with offspring affected by severe HDFN requiring neonatal exchange transfusion and/or intrauterine transfusion. Mothers treated at centers that provided extended antigen negative RBCs (MATCH, 5 centers) and those that do not (NoMATCH, 9 centers) were compared.

Results: 293 mothers had $\geq 1$ affected pregnancy; 179 at MATCH, 114 at NoMATCH centers. Most alloimmunization (83\%) was attributed to previous pregnancy; $3 \%$ to transfusion (2 cases at MATCH, 6 at NoMATCH centers); 14\% was undetermined (both antecedent transfusion and pregnancy). Only 50 mothers had been transfused; 13 had HDFN due to anti-K at MATCH and 4 at NoMATCH centers. Most $(12 / 13,92 \%)$ of the anti-K HDFN cases at MATCH centers had $\mathrm{K}+$ paternal antigen status. Mothers at the MATCH centers do not appear to be protected from HDFN due to $\mathrm{K}, \mathrm{C}, \mathrm{C}$, and $\mathrm{E}$ antibodies, although the low number of transfused FCPs precluded drawing firm conclusions.

Conclusion: The causal stimulus of antibodies that cause HDFN is predominantly from previous pregnancy. Although extended RBC matching for FCPs may impart some protection from allosensitization, we were unable to show a positive effect, possibly because matching policies are not uniform and there was a small number of previously transfused mothers.

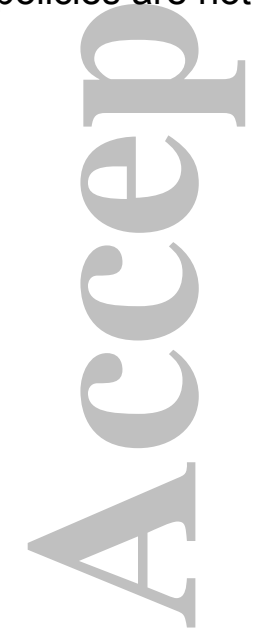




\section{INTRODUCTION}

There is no universally accepted approach for selection of RBC units for transfusion in females of childbearing potential (FCPs). Some centers aim to prevent RBC alloimmunzation to non-RhD RBC antigens, and thereby decrease the risk of hemolytic disease of the fetus and newborn (HDFN) in a future pregnancy by using extended antigen matched (or antigen negative) RBC units for transfusion ${ }^{1}$. Typically this involves $\mathrm{K}$ and/or extended Rh antigen(s) matching, such as $\mathrm{C}, \mathrm{E}$ or $\mathrm{C}$.

It is important to investigate if extended RBC matching is effective in reducing alloimmunization in FCPs. However, because healthy FCPs are not chronically transfused, the rationale for prophylactic $\mathrm{RBC}$ antigen matching may be less compelling. On the other hand, because some cases of HDFN, particularly fetuses with hydrops, have serious clinical sequelae and/or may require significant resources to manage, prevention measures may exert a meaningful positive effect on the population of FCPs that are at risk for developing RBC alloimmunization $^{2-4}$.

The goal of this study was to determine if blood bank policy aimed at decreasing the risk of non-RhD alloimmunization in FCPs by prospective antigen matching is effective. To determine this, the causal stimulus for RBC alloimmunization (previous pregnancy or transfusion) in women with HDFN is needed to enumerate the population available to be protected by a transfusion matching policy. Further, the impact of patients receiving transfusions at more than one center may disrupt the protective effects of a regional matching policy. Together these data can provide novel information about the relative proportion of HDFN that is preventable by prospective RBC transfusion matching policies for FCPs. This pragmatic information will be useful for transfusion medicine policy makers who wish to enact blood bank practices to decrease the risk of future HDFN.

\section{METHODS}

The study was a multi-center retrospective study of women who had at least one pregnancy complicated by severe HDFN. The study was approved by each site's human subjects review board. The inclusion criteria for each mother was to either have had a pregnancy with a fetus that required intrauterine transfusion (IUT), and/or given birth to an infant that required neonatal exchange transfusion (NEX) between January 1, 2000 and December 31, 2012. Mothers were excluded if the IUT and/or NEX was required for causes other than RBC alloimmunization and if the mother was receiving extended matched RBC transfusions for any reason, such as sickle cell disease. 
Demographic data were collected about each participating hospital or blood center and each of the mothers (subjects) entered into the study, including the number of pregnancies and outcome of each pregnancy. Details of all of the subjects' pregnancies were collected, including any interventions needed to treat the fetus or infant affected by HDFN by medical record review at each participating institution. The transfusion and pregnancy histories of FCPs who had at least one pregnancy complicated by severe HDFN were investigated to determine the etiology of the causative antibody. The etiology of the RBC antibody that caused HDFN was defined as follows: 1) transfusion related antibody: Transfusion preceded HDFN and there was no history of a prior pregnancy; 2) pregnancy related: Transfusion did not precede antibody detection and a there was a history of a previous pregnancy; 3) unable to discern: Neither a documented transfusion nor a previous pregnancy preceded the index case of HDFN, or, the transfusion was administered in the setting of a post-partum hemorrhage making it difficult to establish the antibody's origin. If medical record review revealed that a transfusion attributed to a MATCH center's subject was found to have potentially been administered outside of the MATCH center's catchment area, the transfusion was still attributed to the MATCH center. This attribution was made because this study is an analysis of the real world application of matching policies by transfusion services, or stated another way, an "intention to match" analysis. The paternal antigen status was collected if available; data on different fathers was not collected. Data were individually collected at each site, de-identified, and then submitted for analysis using an electronic, secure online case report form system (RedCap, www.redcap.org).

Descriptive statistics were used to summarize the transfusion data. Transfusion episodes were defined by the indication for transfusion exposure, or during one hospitalization regardless of the number of units transfused (e.g. receipt of $4 \mathrm{RBCs}$ following a trauma is considered one transfusion episode, whereas receipt of a single unit of RBCs during 2 separate hospital admissions would have been considered 2 transfusion episodes). In the transfused subjects, the number of HDFN cases that were caused by each specific RBC antigen that was included in a MATCH policy (K, C, E, c) was compared to the transfused subjects who were from NoMATCH centers using a chi-squared analysis to calculate the odds ratio (OR) and $95 \%$ confidence interval..

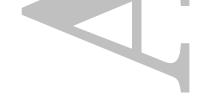

\section{RESULTS}

There were 14 participating centers from seven different nations. Centers ranged in size from 1000 - 100,000 RBC transfusions provided annually. The centers were classified as blood center and immunohematology testing laboratory that supports more than one hospital with 
obstetrical services $(n=1)$, blood center and immunohematology testing laboratory that supports one hospital with obstetrical services $(n=2)$, and hospitals with obstetrical care $(n=11)$ (Table 1). Centers practiced a mix of approaches to transfusion of FCPs; there were $5 \mathrm{MATCH}$ and 9 NoMATCH centers. Within the MATCH centers, different protocols for prophylactic antigen matching for FCPs were employed (Table 1). The RBC antigens that were matched for were in the Rh and Kell blood group systems and are generally considered the most immunogenic and the most frequent cause of HDFN, although the specific reason each antigen was chosen at each of the MATCH centers was not collected. The five MATCH centers were located in the United Kingdom, Sweden, the Netherlands, Switzerland and Australia. The nine NoMATCH centers were in Canada $(n=1)$ and the United States $(n=8)$.

Overall there were 293 FCP subjects that met all of the study inclusion criteria. There were 10 potential subjects with a total of 28 pregnancies at $\mathrm{NoMATCH}$ centers that were excluded due to receipt of extended antigen matched RBCs to treat their hemoglobinopathies. Subject demographics at the MATCH and NoMATCH groups did not differ significantly. There were 50 transfused subjects who had a median of 1 transfusion episode (range $1-7$ ). Attempts were made to determine the indication and timing of the transfusion episode, however, if a patient was not transfused at the participating center and did not give details of a past transfusion to their health care provider, this information was missing from the reviewed medical records and could not be used. The indications for transfusion varied; the most common indication was obstetrical hemorrhage (Table 2).

There were a total of 534 pregnancies amongst the 179 FCPs at the MATCH centers and 301 pregnancies amongst the 114 FCPs at the NoMATCH centers, for a total of 835 pregnancies in this study. Fifty one percent of the 835 pregnancies were affected by severe HDFN. Overall, there was $\geq 90 \%$ survival of infants born to these mothers; a broad range of clinical interventions were used to treat HDFN (Table 3). The affected pregnancies were distributed between MATCH and $\mathrm{NoMATCH}$ centers in virtually identical proportions; $51 \%$ of pregnancies in the subjects at MATCH centers were affected with HDFN (272/534), and 51\% of pregnancies in the subjects at NoMATCH centers were affected with HDFN (153/301, p=1.0, Fisher's exact test).

Medical record review to determine the source of RBC sensitization found that overall $243 / 293$ (83\%) of the subjects were sensitized due to a previous pregnancy (146 from MATCH centers, 97 from NoMATCH centers). For 42/293 (14\%) of the subjects, the etiology of the RBC sensitization could not be determined because the medical records reflected previous pregnancy and previous transfusion (31 from MATCH centers, 11 from NoMATCH centers). Three percent $(8 / 293)$ of the subjects were sensitized due to transfusion (2 from MATCH 
centers, 6 from NoMATCH centers, Table 4, Figure 1). Subjects were alloimmunized to many different RBC antigens (Table 5). An interesting observation is the 12 subjects who had intravenous drug use as a risk factor for HDFN reported in this study. Further details were not available for these subjects.

Of the 50 transfused FCPs, 17 were sensitized to the $\mathrm{K}$ antigen; $13 / 17$ (76\%) at MATCH centers and $4 / 17(24 \%)$ at NoMATCH centers, Table $6.92 \%(12 / 13)$ of the K-sensitized, transfused subjects that were from MATCH centers had a partner (paternal) RBC antigen status that was K-positive, suggesting the causal stimulus was not transfusion. Considering the transfused subjects whose partners (fathers) were known to be $\mathrm{K}$ antigen negative, the numbers attributed to transfusion are too small to draw any conclusions and similar between centers (1 from MATCH and 2 from NoMatch). Thus, the number of patients available to be protected by a transfusion policy of prospective K-antigen matching was so small thatmatching for the $\mathrm{K}$ antigen did not appear to confer protection from K-sensitization (OR 3.00, 95\% Cl: 0.12- 73.64, data not shown). The findings were similar for the other blood group antigens that were included in matching algorithms (E, C, c). Interestingly, 17/35 (49\%) of the transfused subjects at the MATCH centers were noted to have received transfusions outside of the MATCH center's catchment areas, suggesting that the inciting sensitization event could have been from a transfusion episode occurring elsewhere, possibly before emigration to the MATCH center's catchment area. For MATCH centers that match for Rh blood group antigens, the policy was not found to provide protection from alloimmunization (Table 6).

\section{DISCUSSION}

This is the largest international cohort of HDFN cases in which the etiology of the implicated RBC antibody was determined. A very large proportion of RBC antibodies that cause clinically significant HDFN, defined as need for IUT or NEX, were due to previous pregnancy $(83 \%)$. Only $3 \%$ of RBC antibodies in this study were attributed to previous transfusions; however, in $14 \%$ of cases, the etiology of the RBC antibody could not be determined. Given the large number of subjects who were sensitized by a previous pregnancy $(83 \%)$, it is not surprising that extended antigen matching transfusion policies (MATCH) were not found to substantially reduce clinically significant HDFN in this cohort. Another important finding is that a large proportion of transfused subjects at MATCH centers (49\%) were transfused in regions not covered by the matching policy. Although this makes drawing conclusions about the strict utility of matching transfusions for FCPs difficult, it provides 'real world' evidence that patients do not 
seek medical care in a single region during their lifetime, thus potentially defeating the efforts to prevent alloimmunization at the MATCH centers. Moreover, the results provide a broad multiinstitution experience that on a population basis, most patients that will go on to have clinically significant HDFN are not impacted by extended matching transfusion policies because the stimulus for the RBC alloimmunization is either from pregnancy or RBC transfusion received at another institution that does not provide matched RBCs.

A growing body of literature suggests that extended RBC antigen matching to prevent $\mathrm{RBC}$ alloimmunization is advantageous in the setting of hemoglobinopathy patients requiring chronic transfusions, and to reduce the probability of a hemolytic reaction in those with warm autoantibodies. The goal of RBC selection protocols in SCD patients is to prevent alloimmunization in patients that are at risk for future delayed hemolytic transfusion reactions, difficulty in finding compatible blood and rarely, hyperhemolysis reactions ${ }^{5}$. In patients with warm reacting autoantibodies, the purpose of extended RBC matching is to obviate the need for repeat adsorption procedures and potentially decrease the risk of alloimmunization ${ }^{6}$. In this retrospective study, the target population of RBC matching was all females of child-bearing potential, usually defined as those women $<45-50$ years of age. The subset of FCPs that may end up benefitting from RBC matching are those that go on to reproduce, and thus would be likely to be young and healthy, and at low risk for multiple transfusions. These data suggest that RBC matching has a relatively low impact on reducing alloimmunization as only between 3-17\% of the antibodies implicated in causing severe HDFN in this study were, or could have been, caused by transfusion. However, although many cases of HDFN are clinically mild, a percentage of cases do have serious clinical outcomes ${ }^{3,7}$. The goal of prevention of RBC alloimmunization is thus worthwhile, since maternal alloimmunization can result in loss of the pregnancy and devastating neurological morbidity ${ }^{8,9}$.

Several nations routinely provide extended matching for antigens such as $\mathrm{K}, \mathrm{C}, \mathrm{c}$ and $\mathrm{E}$ when transfusing $\mathrm{FCPs}^{10}$. In a retrospective review in Croatia, $48 \%$ of 214 pregnancies with maternal sensitization to $\mathrm{E}, \mathrm{K}$, or $\mathrm{c}$ had a history of transfusion, suggesting that matching for these antigens may be protective, however, the paternal antigen status was not reported ${ }^{11}$. In the current study, the rate of clinically significant RBC sensitization did not differ between MATCH and NoMATCH centers. The low number of transfused subjects precluded finding statistically significant differences between the impact of a MATCH approach when transfused FCPs were cared for at MATCH policy centers (and had not received transfusions elsewhere) and when the father was not RBC antigen positive. Any policy executed from the perspective of the transfusion service laboratory will broadly cover all female patients, regardless of previous 
transfusion history and the RBC phenotype of potential future partners, since this information is not available at the time of blood product selection, especially in emergencies.

A notable finding is the fact that $17 / 35(49 \%)$ transfusion events in subjects included at the MATCH centers occurred in a location that was not covered by the center's transfusion policy. This suggests that transfusion during travel or before emigration to the catchment area of a MATCH center may play a significant role in the sensitization found in this study.

A major limitation is the size of the AMIGO study population; in particular there were relatively few FCPs that had significant HDFN who were transfused (17\%). Because HDFN is a rare disease and the inciting sensitizing event usually occurs well before the affected pregnancy, we wished to have as large of a study population as possible. Consequently 14 centers in seven countries participated, yet, the final subject numbers were not large enough to conclude that RBC antigen matching impacted the development of HDFN. It is possible that the rate of transfusion related HDFN is higher than reported here due to selection of subjects that had severe enough courses that required NEX or IUT. Moreover, the small numbers of Rh sensitized mothers, i.e., those with anti-C, -E, -C, cannot support or refute the practice of prospective antigen matching. The study, however, is still the largest cohort published to date in which antibody derivation and transfusion history was intensively investigated, suggesting that the rate of transfusion and proportion of fathers who are RBC antigen positive is generalizable. Another limitation of the study is the retrospective medical record review which may have omissions that could result in assignment of FCPs to incorrect study groups (transfused, not transfused). This could be improved with a prospective study design, or using the database of a nationalized healthcare system. However, prospective study of such a rare event (HDFN) would be difficult to accomplish, given the low overall numbers found in AMIGO, even when using a study time frame of $>10$ years and a large number of study centers. Lastly, the study did not specifically identify intrauterine transfusion, as a subset of the transfusion episodes, as a cause for sensitization, a known risk for additional antibody formation ${ }^{12}$.

To implement any prospective RBC matching policy that is likely to impact patient RBC alloimmunization, broad application across many (or all) transfusing facilities in an area would be needed, particularly given the finding that a significant number of transfusions occurred away from the subject's primary treatment center, or the hospital where they actually gave birth. In SCD patients in the United States, facilities with prospective matching policies note RBC alloantibodies presumed to have been acquired following transfusion at a facility outside of their

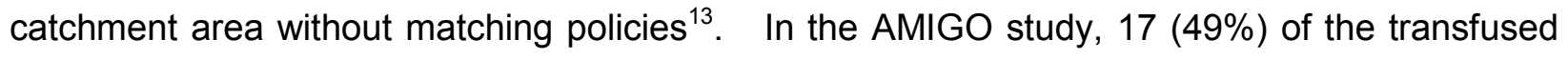
FCPs from MATCH centers received their transfusion(s) in regions outside of the MATCH center's catchment area. This highlights the difficulty of realizing success from transfusion 
policies meant to offer prospective protection from RBC alloimmunization in the setting of regional or global travel and immigration.

With the goal of continuous improvement in patient transfusion care, many blood collectors are working to provide RBC antigen typing/genotyping data on RBC units to transfusion services to improve their ability to find blood for patients with RBC antibodies. The collectors are using serology and/or RBC genotyping methods to RBC antigen type blood donors $^{14-16}$. As the stocks of antigen typed RBC units increase due to these activities, the availability to provide extended antigen matched RBCs will improve. In the future, easier access to RBC units may make broad matching policy changes more feasible. To determine the best transfusion policies for FCPs, the transfusion medicine community must continue to weigh the travel patterns of their transfused population, and the availability of antigen typed RBC units against the potential impact of matching FCPs in their own catchment area. Strict adherence to restrictive transfusion policies may also lead to decreased RBC alloimmunization, and decreased HDFN, in the minority of HDFN cases that are caused by blood transfusion.

\section{ACKNOWLEDGEMENTS}

We would like to thank Eileen Selogie for her support of the design and maintenance of the data collection tools. We would also like to thank Rebecca Barty. Many of the authors are scientific members and guests of the Biomedical Excellence for Safer Transfusion (BEST) Collaborative, an international research organization that works collaboratively to improve transfusion-related services through standardization of analytic techniques, development of new procedures, systematic review of evidence, and execution of clinical and laboratory studies. 


\section{TABLES \& FIGURES}

Table 1. Center demographics and details of RBC antigen matching policies for FCPsFCPs at MATCH centers. $(n=14)$

\begin{tabular}{|c|c|c|}
\hline & $\begin{array}{c}\text { MATCH } \\
\text { centers } \\
(n=5)\end{array}$ & $\begin{array}{c}\text { NoMATCH } \\
\text { centers } \\
(n=9)\end{array}$ \\
\hline \multicolumn{3}{|l|}{ Center type } \\
\hline $\begin{array}{l}\text { Blood supplier, Testing service that supports }>1 \text { hospital with } \\
\text { obstetrical care }\end{array}$ & 1 & 0 \\
\hline $\begin{array}{l}\text { Blood supplier, Testing service that supports } 1 \text { hospital with } \\
\text { obstetrical care }\end{array}$ & 1 & 1 \\
\hline Hospital that supports obstetrical care & 3 & 8 \\
\hline \multicolumn{3}{|l|}{ Center size: Number of deliveries / year } \\
\hline$\leq 5,000$ & 2 & 6 \\
\hline $5,001-10,000$ & 2 & 2 \\
\hline $10,001-30,000$ & 1 & 1 \\
\hline \multicolumn{3}{|l|}{ Center size: Number of RBC transfusions / year } \\
\hline$\leq 10,000$ & 1 & 4 \\
\hline $10,001-50,000$ & 3 & 5 \\
\hline $50,001-100,000$ & 1 & 0 \\
\hline \multicolumn{3}{|l|}{ MATCH Centers: Prophylactic matching protocol } \\
\hline - $K$ antigen for females $<50-60$ years & 2 & \\
\hline - $\mathrm{K}, \mathrm{C}$ antigens for females for $<50$ years & 1 & \\
\hline - $\mathrm{K}, \mathrm{C}, \mathrm{C}, \mathrm{E}, \mathrm{e}$ antigens for females $<50$ years & 1 & \\
\hline - $\mathrm{K}, \mathrm{C}, \mathrm{E}$ antigens for females $<45$ years & 1 & \\
\hline
\end{tabular}


Table 2. Demographic, obstetric and transfusion demographics of FCPs by MATCH and NoMATCH centers ( $n=293$ included FCPs).

\begin{tabular}{|c|c|c|}
\hline FCP demographics & MATCH & NoMATCH \\
\hline FCP, total number ( $\%$ of total number of FCPs) & $179(61 \%)$ & $114(39 \%)$ \\
\hline FCP Age (y), mean (range) & $33.1(19-46)$ & $31.1(20-46)$ \\
\hline \multicolumn{3}{|l|}{ Ethnicity, number (\% of total number of FCPs) } \\
\hline - Black & $6(2 \%)$ & $18(6 \%)$ \\
\hline - Asian, Polynesian & $5(2 \%)$ & 0 \\
\hline - Caucasian & $141(48 \%)$ & $71(24 \%)$ \\
\hline - Hispanic, Latina & $1(0 \%)$ & $7(2 \%)$ \\
\hline - Native American, Inuit & $1(0 \%)$ & $1(0 \%)$ \\
\hline - Other & $25(9 \%)$ & $17(6 \%)$ \\
\hline Number of pregnancies per FCP, median (range) & $3(1-11)$ & $2(1-11)$ \\
\hline $\begin{array}{l}\text { Number of transfused FCPs, FCPs ( } \% \text { of total number of FCPs) } \\
\text { (total number of transfused FCPs }=50 \text { ) }\end{array}$ & $33(11 \%)$ & $17(6 \%)$ \\
\hline \multicolumn{3}{|c|}{ Number of FCPs transfused by indication (number of transfusion episodes) ${ }^{\star \Lambda}$} \\
\hline - Obstetrical hemorrhage & $24(24)$ & $6(8)$ \\
\hline - Unknown, other & $5(6)$ & $7(8)$ \\
\hline - Trauma & $2(2)$ & $2(2)$ \\
\hline - Surgery, non-trauma related & $2(2)$ & $2(2)$ \\
\hline - Cancer & $1(1)$ & 0 \\
\hline - Non-malignant hematological disease & 0 & $1(7)$ \\
\hline - Anemia & $1(1)$ & $0(0)$ \\
\hline FCP received platelet transfusion in isolation from $\mathrm{RBC}$ & 0 & $7(2)$ \\
\hline FCP with history of IVDU & $2(1)$ & $10(3)$ \\
\hline
\end{tabular}

* There were 63 distinct transfusion episodes amongst50 FCPs. Transfusion episodes are defined by indication for transfusion, or during one hospitalization (e.g. receipt of 4 RBCs following a trauma is considered one transfusion episode). Three of the subjects with obstetrical hemorrhage also had another transfusion episode with a different indication.

${ }^{\wedge} 49 \%$ of transfused MATCH center subjects (17 subjects) occurred in a region that is not covered by the MATCH center's transfusion policy (e.g. transfusion in another nation before the FCP immigrated to a nation where the MATCH center provided care).

HDFN: hemolytic disease of the fetus and newborn; IVDU: intravenous drug use 
Table 3. Outcomes and interventions for each affected pregnancy, fetus and/or infant due to HDFN.

\begin{tabular}{|c|c|c|}
\hline & MATCH & NOMATCH \\
\hline Number of FCPs & 179 & 114 \\
\hline Total number of pregnancies & 534 & 301 \\
\hline $\begin{array}{l}\text { Number of pregnancies affected by severe HDFN , \% of } \\
\text { pregnancies affected by HDFN (total } n=425 \text { ) }\end{array}$ & $272(51 \%)$ & $153(51 \%)$ \\
\hline \multicolumn{3}{|l|}{ Fate of each pregnancy affected by severe HDFN, n (\%) } \\
\hline - Term infant (>37 weeks), live born, survived & 107 & 67 \\
\hline - Pre-term infant (<37 weeks), live born, survived & 148 & 70 \\
\hline - Pre-term infant (<37 weeks), live born, expired & 4 & 6 \\
\hline - Still birth & 5 & 3 \\
\hline - Miscarriage & 3 & 1 \\
\hline - Unknown & 5 & 6 \\
\hline Overall survival for infant affected by severe HDFN & $\begin{array}{l}255 / 272 \\
(94 \%)\end{array}$ & $137 / 153(90 \%)$ \\
\hline \multicolumn{3}{|l|}{ Clinical findings and interventions } \\
\hline - Intrauterine transfusions, total (IUT) & 169 & 109 \\
\hline - IUT per pregnancy per enrolled mother & 2.75 & 3.15 \\
\hline - Hyperbilirubinemia, no treatment needed & 5 & 2 \\
\hline - Phototherapy & 81 & 56 \\
\hline - Simple RBC transfusion & 27 & 39 \\
\hline - Exchange transfusion & 57 & 57 \\
\hline - IVlg & 0 & 32 \\
\hline - Unknown, other & 17 & 28 \\
\hline
\end{tabular}

HDFN: hemolytic disease of the fetus and newborn, * Includes term \& pre-term infants who survived; does not include fetal interventions such as IUT.

Table 4. See below (Landscape orientation) 
Table 5. Causative maternal RBC antibodies implicated in 425 pregnancies affected by HDFN

\begin{tabular}{|c|c|c|c|c|c|c|}
\hline \multirow{2}{*}{$\begin{array}{l}\text { Antibody } \\
\text { Specificity }\end{array}$} & \multicolumn{2}{|c|}{ Total Number } & \multicolumn{2}{|c|}{ MATCH Centers } & \multicolumn{2}{|c|}{ NoMATCH Centers } \\
\hline & Number & $\begin{array}{c}\% \text { of all } \\
\text { antibodies }\end{array}$ & Number & $\begin{array}{c}\% \text { of all } \\
\text { antibodies }\end{array}$ & Number & $\begin{array}{c}\% \text { of all } \\
\text { antibodies }\end{array}$ \\
\hline $\mathrm{D}$ & 279 & $41.2 \%$ & 171 & $25.2 \%$ & 108 & $15.9 \%$ \\
\hline C & 133 & $19.6 \%$ & 70 & $10.3 \%$ & 63 & $9.3 \%$ \\
\hline $\mathrm{K}$ & 78 & $11.5 \%$ & 61 & $9.0 \%$ & 17 & $2.5 \%$ \\
\hline$E$ & 65 & $9.6 \%$ & 35 & $5.2 \%$ & 30 & $4.4 \%$ \\
\hline $\mathrm{C}$ & 50 & $7.4 \%$ & 33 & $4.9 \%$ & 17 & $2.5 \%$ \\
\hline $\mathrm{Jk}^{\mathrm{a}}$ & 19 & $2.8 \%$ & 9 & $1.3 \%$ & 10 & $1.5 \%$ \\
\hline $\mathrm{Jk}^{\mathrm{b}}$ & 7 & $1.0 \%$ & 3 & $0.4 \%$ & 4 & $0.6 \%$ \\
\hline$S$ & 7 & $1.0 \%$ & 1 & $0.1 \%$ & 6 & $0.9 \%$ \\
\hline $\mathrm{Fy}^{\mathrm{a}}$ & 4 & $0.6 \%$ & 3 & $0.4 \%$ & 1 & $0.1 \%$ \\
\hline s & 1 & $0.1 \%$ & 0 & $0.0 \%$ & 1 & $0.1 \%$ \\
\hline $\mathrm{Fy}^{\mathrm{b}}$ & 1 & $0.1 \%$ & 0 & $0.0 \%$ & 1 & $0.1 \%$ \\
\hline $\begin{array}{c}\mathrm{Yt}^{\mathrm{a}}, \mathrm{Yt}^{\mathrm{b}}, \\
\mathrm{Lea}, \mathrm{V}, \mathrm{Cw}, \\
\mathrm{Wr} \mathrm{r}^{\mathrm{a}}, \mathrm{U}, \text { etc. }\end{array}$ & 34 & $5.0 \%$ & 15 & $2.2 \%$ & 19 & $2.8 \%$ \\
\hline
\end{tabular}




\section{Figure 1.}

Transfusion and paternal antigen status patterns in subjects at MATCH and NoMATCH centers. The father was antigen positive in $94 \%$ vs $55 \%$ of subjects MATCH and $\mathrm{NoMATCH}$, respectively.

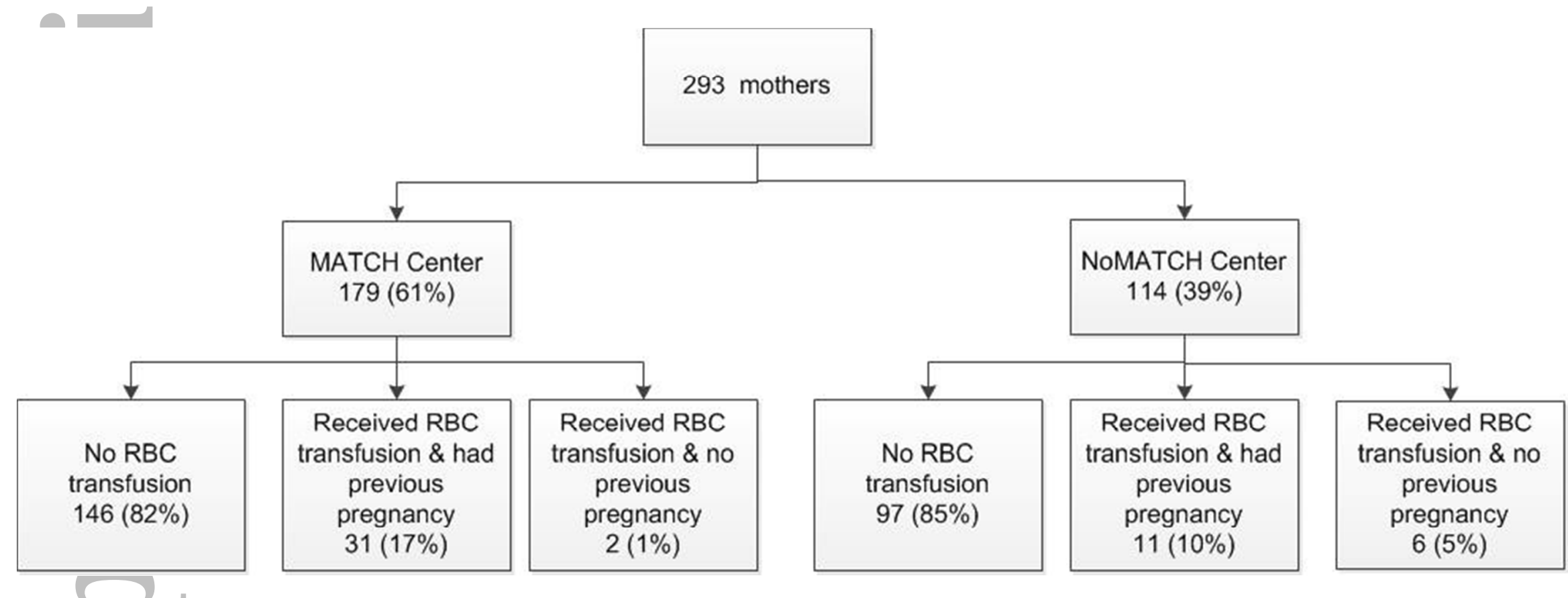

Legend: The etiology of the RBC antibody that caused HDFN was defined as follows: 1) transfusion related antibody: Transfusion preceded HDFN and there was no history of a prior pregnancy; 2) pregnancy related: Transfusion did not precede antibody detection and a there was a history of a previous pregnancy; 3 ) unable to discern: Neither a documented transfusion nor a previous pregnancy preceded the index case of HDFN, or, the transfusion was administered in the setting of a post-partum hemorrhage making it difficult to establish the antibody's origin. The father's antigen status was also collected when possible.

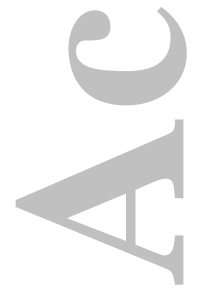


Table 4. Case histories for the eight women with HDFN likely to have been caused by RBC transfusion.

\begin{tabular}{|c|c|c|c|c|c|c|}
\hline Case & $\begin{array}{c}\text { RBC } \\
\text { Antibody(ies) }\end{array}$ & $\begin{array}{c}\text { Number of } \\
\text { Transfusion } \\
\text { Episodes }\end{array}$ & $\begin{array}{c}\text { RBC Transfusion } \\
\text { Indication }\end{array}$ & $\begin{array}{c}\text { MATCH or } \\
\text { NoMATCH } \\
\text { Center }\end{array}$ & $\begin{array}{c}\text { NEX } \\
\text { and/or IUT }\end{array}$ & Pregnancy Outcome \\
\hline 1 & $\mathrm{~K}, \mathrm{c}, \mathrm{E}$ & 2 & Other & NoMATCH & NEX & Term baby, survived \\
\hline 2 & $\mathrm{~K}$ & 1 & Unknown & MATCH & NEX, IUT & Term baby, survived \\
\hline 3 & $\mathrm{c}$ & 1 & Obstetrical hemorrhage & MATCH & NEX, IUT & Pre term baby, survived \\
\hline 4 & $\mathrm{E}, \mathrm{G}$ & 1 & Obstetrical hemorrhage & NoMATCH & NEX, IUT & Pre term baby, survived \\
\hline 5 & $\mathrm{~K}, \mathrm{C}$ & 1 & Trauma, MVA & NoMATCH & IUT & Stillbirth \\
\hline 6 & $\mathrm{D}, \mathrm{Wr}^{\mathrm{a}}$ & 1 & Unknown & NoMATCH & IUT & Term baby, survived \\
\hline 7 & $\mathrm{C}, \mathrm{Jk}$ & 1 & Unknown & NoMATCH & IUT & Term baby, survived \\
\hline 8 & $\mathrm{D}, \mathrm{C}, \mathrm{G}$ & 1 & Trauma, MVA & NoMATCH & NEX, IUT & Pre term baby, survived \\
\hline
\end{tabular}

MVA = motor vehicle accident, IUT=intrauterine transfusion, NEX=neonatal exchange transfusion

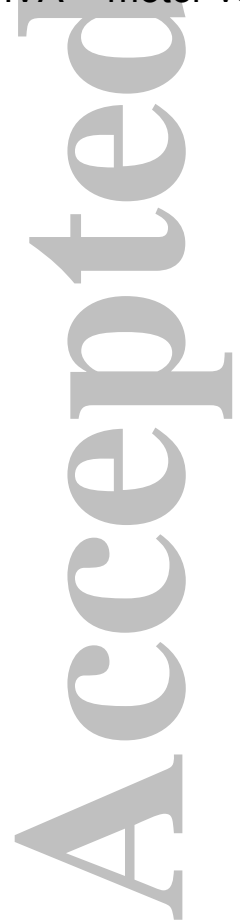




\section{Table 6}

Comparison of MATCH and NoMATCH centers' RBC sensitization rates and antibody specificities in the transfused FCPs ( $\mathrm{n}=50$ ) that had pregnancies affected by HDFN. This table lists only antigens that are matched for at the MATCH centers. There were 33 FCPs that were alloimmunized to at least 1 RBC antigen at the MATCH centers and 17 at the NoMATCH centers.

\begin{tabular}{|c|c|c|c|c|c|}
\hline RBC antibody specificity & \multicolumn{2}{|c|}{ FCP Sensitized? } & MATCH & NoMATCH & OR $[95 \% \mathrm{Cl}]$ \\
\hline \multirow{2}{*}{$\mathrm{K}$} & Yes & 17 & 13 & 4 & \multirow{2}{*}{$2.11[0.56-7.91]$} \\
\hline & No & 33 & 20 & 13 & \\
\hline \multirow{2}{*}{ C } & Yes & 8 & 4 & 4 & \multirow{2}{*}{$3.2[0.67-15.19]$} \\
\hline & No & 42 & 10 & 32 & \\
\hline \multirow{2}{*}{ c } & Yes & 19 & 9 & 10 & \multirow{2}{*}{$1.89[0.58-6.11]$} \\
\hline & No & 31 & 10 & 21 & \\
\hline \multirow{2}{*}{$E$} & Yes & 13 & 5 & 8 & \multirow{2}{*}{$1.03[0.28-3.77]$} \\
\hline & No & 37 & 14 & 23 & \\
\hline
\end{tabular}




\section{REFERENCES}

1. Koelewijn JM, Vrijkotte TG, de Haas M, van der Schoot CE, Bonsel GJ. Risk factors for the presence of non-rhesus D red blood cell antibodies in pregnancy. Bjog 2009;116:655.

2. Higgins JM, Sloan SR. Stochastic modeling of human RBC alloimmunization: evidence for a distinct population of immunologic responders. Blood 2008;112:2546.

3. Smits-Wintjens VE, Rath ME, van Zwet EW, et al. Neonatal morbidity after exchange transfusion for red cell alloimmune hemolytic disease. Neonatology 2013;103:141-147.

4. Lindenburg IT, Smits-Wintjens Ve Fau - van Klink JM, van Klink Jm Fau - Verduin E, et al. Longterm neurodevelopmental outcome after intrauterine transfusion for hemolytic disease of the fetus/newborn: the LOTUS study. Am J Obstet Gynecol 2012; 206;141.e1-8

5. Castro O, Sandler SG, Houston-Yu P, Rana S. Predicting the effect of transfusing only phenotypematched RBCs to patients with sickle cell disease: theoretical and practical implications. Transfusion 2002;42:684.

6. Shirey RS, Boyd JS, Parwani AV, Tanz WS, Ness PM, King KE. Prophylactic antigen-matched donor blood for patients with warm autoantibodies: an algorithm for transfusion management. Transfusion 2002;42:1435.

7. Rath ME, Smits-Wintjens VE, Lindenburg IT, et al. Postnatal outcome in neonates with severe Rhesus c compared to rhesus D hemolytic disease. Transfusion 2013;53:1580-1585.

8. Lindenburg IT, Smits-Wintjens VE, van Klink JM, et al. Long-term neurodevelopmental outcome after intrauterine transfusion for hemolytic disease of the fetus/newborn: the LOTUS study. American journal of obstetrics and gynecology 2012;206:141.e141-148.

9. Lindenburg IT, van Kamp IL, van Zwet EW, Middeldorp JM, Klumper FJ, Oepkes D. Increased perinatal loss after intrauterine transfusion for alloimmune anaemia before 20 weeks of gestation. Bjog 2013;120:847-852.

10. Koelewijn JM, Vrijkotte TG, van der Schoot CE, Bonsel GJ, de Haas M. Effect of screening for red cell antibodies, other than anti-D, to detect hemolytic disease of the fetus and newborn: a population study in the Netherlands. Transfusion 2008;48:941.

11. Dajak S, Culic S, Stefanovic V, Lukacevic J. Relationship between previous maternal transfusions and haemolytic disease of the foetus and newborn mediated by non-RhD antibodies. Blood Transfus 2013;11:528-532.

12. Schonewille H, Klumper FJ, van de Watering LM, Kanhai HH, Brand A. High additional maternal red cell alloimmunization after Rhesus- and K-matched intrauterine intravascular transfusions for hemolytic disease of the fetus. American journal of obstetrics and gynecology 2007;196:143.e141. 13. Harm SK, Yazer MH, Monis GF, Triulzi DJ, Aubuchon JP, Delaney M. A centralized recipient database enhances the serologic safety of RBC transfusions for patients with sickle cell disease. American journal of clinical pathology 2014;141:256.

14. Nance ST. Operational issues for implementation of donor genotyping for red blood cell antigens. Transfusion 2007;47:69s-72s.

15. Flegel WA, Gottschall JL, Denomme GA. Implementing mass-scale red cell genotyping at a blood center. Transfusion 2015;55:2610-2615; quiz 2609.

16. Veldhuisen B, van der Schoot CE, de Haas M. Blood group genotyping: from patient to highthroughput donor screening. Vox sanguinis 2009;97:198-206. 

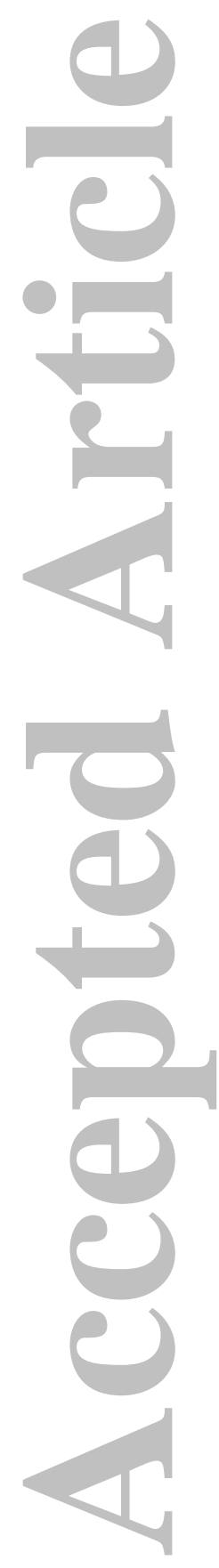

ScholarOne, 375 Greenbrier Drive, Charlottesville, VA, 229011 (434) 964-4100

This article is protected by copyright. All rights reserved. 


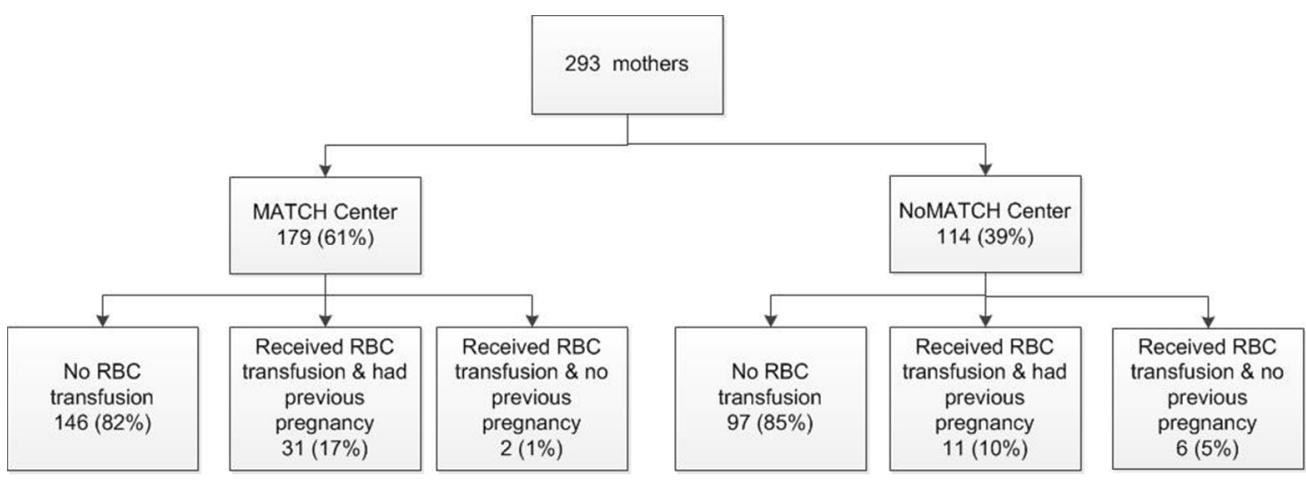

Figure 1

$259 \times 91 \mathrm{~mm}(96 \times 96 \mathrm{DPI})$
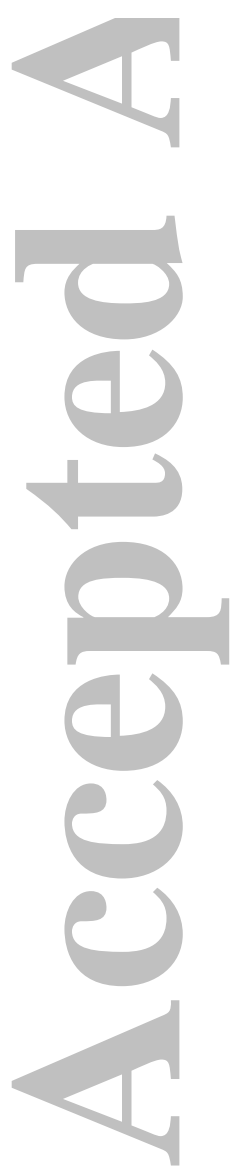

ScholarOne, 375 Greenbrier Drive, Charlottesville, VA, 229011 (434) 964-4100

This article is protected by copyright. All rights reserved. 


\section{University Library}

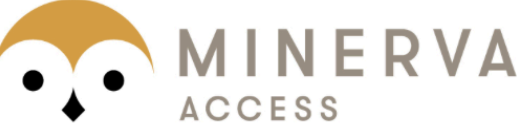

A gateway to Melbourne's research publications

Minerva Access is the Institutional Repository of The University of Melbourne

\section{Author/s:}

Delaney, M;Wikman, A;van de Watering, L;Schonewille, H;Verdoes, JP;Emery, SP;Murphy, MF;Staves, J;Flach, S;Arnold, DM;Kaufman, RM;Ziman, A;Harm, SK;Fung, M;Eppes, CS;Dunbar, NM;Buser, A;Meyer, E;Savoia, H;Abeysinghe, P;Heddle, N;Tinmouth, A;Traore, AN; Yazer, $\mathrm{MH}$

Title:

Blood Group Antigen Matching Influence on Gestational Outcomes (AMIGO) study

Date:

2017-03-01

\section{Citation:}

Delaney, M., Wikman, A., van de Watering, L., Schonewille, H., Verdoes, J. P., Emery, S. P., Murphy, M. F., Staves, J., Flach, S., Arnold, D. M., Kaufman, R. M., Ziman, A., Harm, S. K., Fung, M., Eppes, C. S., Dunbar, N. M., Buser, A., Meyer, E., Savoia, H. ,... Yazer, M. H. (2017). Blood Group Antigen Matching Influence on Gestational Outcomes (AMIGO) study. TRANSFUSION, 57 (3), pp.525-532. https://doi.org/10.1111/trf.13977.

Persistent Link:

http://hdl.handle.net/11343/292414 